\title{
Anonymous Cardinality Estimation in RFID Systems with Multiple Readers
}

\author{
Vahid Shah-Mansouri and Vincent W.S. Wong \\ Department of Electrical and Computer Engineering, \\ The University of British Columbia, Vancouver, Canada \\ e-mail: $\{$ vahids, vincentw $\} @$ ece.ubc.ca
}

\begin{abstract}
In this paper, we study the anonymous cardinality estimation problem in radio frequency identification (RFID) systems. To preserve privacy and anonymity, each tag only transmits a portion of its ID to the reader when it is being queried. To achieve complete system coverage and increase the accuracy of measurement, multiple readers with overlapping interrogation zones are deployed. The cardinality estimation problem is to estimate the total number of tags (or the tag population) in an RFID system. We first propose an exclusive estimator to estimate the number of tags that are exclusively located in the interrogation zone of a selected reader. We then present a multiple-reader tag estimation (MRTE) algorithm that can accurately estimate the tag population using the measurement from different readers and the exclusive estimator. The accuracy of our proposed algorithm and the approximation are validated via simulations. We compare our proposed MRTE algorithm with the enhanced zero-based (EZB) and maximum a posteriori tag estimation (MPTE) algorithms. Although the mean of the estimation error for all three algorithms approaches zero under certain circumstances, the variance of the estimation error for MRTE algorithm increases linearly with the number of readers while it increases exponentially for EZB and MPTE algorithms.
\end{abstract}

\section{INTRODUCTION}

Radio frequency identification (RFID) systems are increasingly being deployed as automated identification systems. These systems are expected to play an important role in various applications such as warehouse and supply chain management, object tracking, patients' monitoring in health care facilities, and security applications. An RFID system consists of a set of readers and several objects, with each object equipped with a small and inexpensive computer chip, called tag. Using these inexpensive tags, every object can be uniquely identified among thousands of objects in an RFID system. Each reader is responsible for interrogation of the tags within its interrogation zone. Since the interrogation range of each reader is limited, several readers are required to fully cover the entire system.

In an RFID system, since the readers and tags share a common wireless channel, tag-to-tag, reader-to tag, and reader-to-reader collisions may occur during an interrogation process. Several anti-collision interrogation techniques have been proposed in the literature [1]-[7]. A framed-slotted ALOHA-based tag anti-collision scheme has also been recently standardized by EPCglobal in [8], which allows each tag to randomly select a time slot and transmit its ID.

In order to preserve privacy and anonymity of the tag users, it may not be necessary to identify each individual user in some RFID applications. Instead, the goal is to estimate the total number of tags (or users) in the system. This is called the cardinality estimation (or tag population) problem in RFID system. In [9], Kodialam et al. proposed zerobased and collision-based tag estimation techniques using a framed-slotted ALOHA model with a single reader. In [10], they extended their work by introducing enhanced-zero based (EZB) estimator which is an asymptotically unbiased estimator. They showed that the EZB algorithm can also be used for RFID systems with multiple readers. In [11], Chen proposed the maximum a posteriori tag estimation (MPTE) technique. Given the number of empty slots and slots with single and collision transmissions in a framed-slotted ALOHA model, the number of tags is estimated as the value which maximizes the a posteriori probability. Other tag estimation techniques have also been proposed in the literature for RFID systems with a single reader [12], [13].

For large scale RFID systems, it is necessary to deploy multiple readers with overlapped interrogation zones in order to fully cover the area and achieve a high accuracy in the estimation. Consequently, a tag can be within the interrogation zone of several readers simultaneously. For tracking applications which require privacy and anonymity of the users, each tag only transmits a portion of its ID to the reader when it is being queried. Thus, readers cannot identify uniquely the individual tags. It is a challenge to estimate the number of tags which are within the interrogation zones of multiple readers.

In this paper, we study the anonymous cardinality estimation problem in an RFID system with multiple readers having overlapping interrogation zones. The contributions are as follows:

- We first propose an exclusive estimator to estimate the number of tags which are only within the interrogation zone of a chosen reader, but not its neighboring readers.

- We propose a multiple-reader tag estimation (MRTE) algorithm which can accurately estimate the tag population using the measurement from different readers and the exclusive estimator. We show that the probability density function (pdf) of the estimation error can be approximated as a normal distribution with zero mean. The accuracy of our proposed algorithm and the approximation is validated via simulations.

- We compare our proposed MRTE algorithm with EZB [10] and MPTE [11] algorithms. Although all three algorithms have a zero mean in estimation error, the variance of the estimation error for MRTE algorithm only increases linearly with the number of readers while it increases exponentially for EZB and MPTE algorithms.

The rest of this paper is organized as follows: The system model is introduced in Section II. In Section III, we first 
propose an exclusive estimator. Then, we propose an MTRE algorithm to estimate the total number of tags in the RFID system and derive the estimation error. Performance evaluation and comparison are presented in Section IV. Conclusions are given in Section V.

\section{SySTEM MODEL}

\section{A. Notations and Model}

Consider an RFID system with multiple readers having overlapping interrogation zones. In the interrogation process, each reader broadcasts a query message which includes the information such as the frame size $f$, persistent probability $p$, and random seed $q$. All tags use the $p$-persistent framed-slotted ALOHA medium access control (MAC) protocol to access the channel. Each tag decides whether or not to transmit in the current frame based on the persistent probability $p$. If a tag decides to transmit, it selects a time slot which is based on a uniform distribution related to its ID, the probability $p$, and seed $q$. Note that given the specific values of the random seed $q$ and persistent probability $p$, the tag selects exactly the same slot in a frame of size $f$, regardless of how many times it has received the query message. To preserve the anonymity and privacy of the user, each tag only transmits part of its ID to the reader in the reply message. Slot synchronization is performed by the reader's query message. All readers use the same seed $q$, probability $p$, and frame size $f$ for each interrogation. The interrogation process of different readers needs not be synchronous. The neighboring readers use time division multiple access (TDMA) technique to prevent readerto-reader collision during the interrogation process.

Let $\mathcal{R}$ denote the set of readers in the system. Let $\mathcal{T}_{r}$ denote the set of tags within the interrogation zone of reader $r \in \mathcal{R}$. Let $n_{r}$ denote the number of tags in the interrogation zone of reader $r \in \mathcal{R}$ (i.e., $\left.n_{r}=\left|\mathcal{T}_{r}\right|\right)$. Let $\mathbf{v}_{r}=\left(v_{r}^{1}, \ldots, v_{r}^{f}\right)$ denote the vector created by reader $r$ after performing an interrogation process, where $v_{r}^{l}$ (with $l=1, \ldots, f$ ) indicates whether the $l^{\text {th }}$ time slot is empty (i.e., $v_{r}^{l}=0$ ) or has at least one transmission (i.e., $v_{r}^{l}=1$ ). The number of elements of vector $\mathbf{v}_{r}$ is equal to the frame size $f$. Fig. 1 shows a two-reader RFID system with overlapped interrogation zones and the interrogation vectors.

\section{B. Estimation of Number of Tags from a Single Reader}

We used the EZB estimator in [10] to estimate the number of tags within the zone of a single reader. EZB used frame-slotted ALOHA MAC and the interrogation vector for estimation. Given the frame size $f$, the persistent probability $p$, and the number of empty slots in $\mathbf{v}_{r}$ after performing an interrogation process $x_{r}$, the EZB estimator approximates $n_{r}$ as

$$
\tilde{n}_{r}=\frac{f}{p} \ln \left(\frac{f}{x_{r}}\right), \quad r \in \mathcal{R}
$$

It can be shown that the mean and variance of the EZB estimator can be approximated as [10, Theorem 3]

$$
\mu_{\mathrm{EZB}}=n_{r}+\frac{e^{p n_{r} / f}-\left(1+p^{2} n_{r} / f\right)}{2 p^{2}},
$$

and

$$
\sigma_{\mathrm{EZB}}^{2}=\frac{f\left(e^{p n_{r} / f}-\left(1+p^{2} n_{r} / f\right)\right)}{p^{2}} .
$$

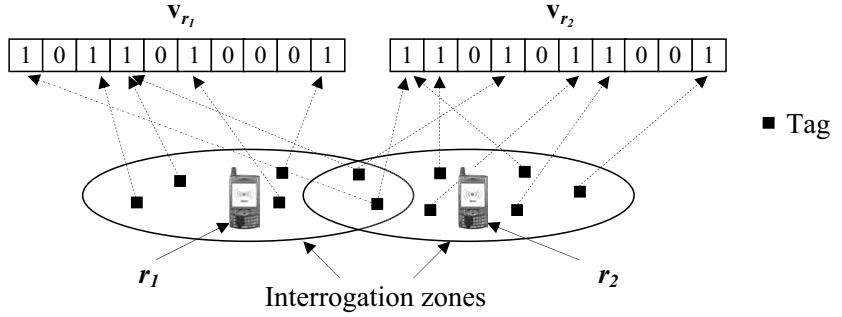

Fig. 1. An RFID system with two readers $r_{1}$ and $r_{2} . n_{r_{1}}=6, n_{r_{2}}=7$, $N=11, f=10$, and $p=1$.

The EZB estimator can estimate the number of tags within the interrogation zone of a reader. However, it cannot estimate the number of tags which is only within the interrogation zone of a particular reader but not within the zones of neighboring readers. In the next section, we present an exclusive estimator and the MRTE algorithm which can estimate the number of tags exclusively located within the interrogation zone of a reader and the total number of tags in the system, respectively.

\section{PROBlem Formulation}

In this paper, the goal is to estimate the total number of tags in a field covered by multiple RFID readers. To obtain an accurate estimate of the total number of tags in the system, the number of tags in the overlapped interrogation zones is required in addition to the approximate number of tags in the interrogation zone of each reader. Consider an RFID system in Fig. 1 with two readers $r_{1}$ and $r_{2}$, with an overlapped interrogation zone. The total number of tags in the system is equal to either $\left(\left|\mathcal{T}_{1}\right|+\left|\mathcal{T}_{2} \backslash \mathcal{T}_{1}\right|=6+5=11\right)$ or $\left(\left|\mathcal{T}_{2}\right|+\left|\mathcal{T}_{1} \backslash \mathcal{T}_{2}\right|=7+4=11\right)$. In general, for $m$ readers $r_{1}, \ldots, r_{m} \in \mathcal{R}$ with overlapping interrogation zones, the total number of tags $N$ in the system is

$$
N=\left|\mathcal{T}_{r_{1}}\right|+\left|\mathcal{T}_{r_{2}} \backslash \mathcal{T}_{r_{1}}\right|+\cdots+\left|\mathcal{T}_{r_{m}} \backslash\left\{\mathcal{T}_{r_{1}} \cup \cdots \cup \mathcal{T}_{r_{m-1}}\right\}\right| .
$$

Note that the order chosen to calculate $N$ has no effect on the final result. Let $\mathcal{H}_{r}$ denote the set of neighboring readers of reader $r \in \mathcal{R}$. Let $\mathcal{W}$ denote a subset of the set of neighbors of reader $r$ (i.e., $\mathcal{W} \subseteq \mathcal{H}_{r}$ ).

Let $n_{r(\mathcal{W})}=\left|\mathcal{T}_{r} \backslash\left\{\cup_{w \in \mathcal{W}} \mathcal{T}_{w}\right\}\right|$ denote the number of tags that are within the zone of reader $r$ but not in the zones of neighboring readers in $\mathcal{W}$. Equation (4) can be used to find the total number of tags $N$ in the system. In the next subsection, we propose an exclusive estimator to calculate the value of $n_{r(\mathcal{W})}$ having the vectors $\mathbf{v}_{r}$ and $\mathbf{v}_{w}$ for $w \in \mathcal{W}$. Then, we present the MRTE algorithm to calculate the total number of tags $N$ using an exclusive estimator.

\section{A. Exclusive Estimator}

As mentioned earlier, whenever a tag is given a random seed $q$ and probability $p$, it selects the same time slot in a frame of size $f$ to transmit. Let $Z$ denote the number of time slots which are nonempty in vector $\mathbf{v}_{r}$, but are empty in vector $\mathbf{v}_{w}, \forall w \in \mathcal{W}$. That is, given reader $r \in \mathcal{R}$, we have

$$
Z=\sum_{l=1}^{f} v_{r}^{l} \prod_{w \in \mathcal{W}}\left(1-v_{w}^{l}\right)
$$


For a given time slot $l$, which is non-empty in $\mathbf{v}_{r}$ (i.e., $v_{r}^{l}=1$ ) but is empty in vector $\mathbf{v}_{w}$ (i.e., $v_{w}^{l}=0$ ), it implies that at least one tag which is within the interrogation zone of reader $r$ has chosen this time slot and none of the tags which are also within the interrogation zone of neighboring readers $w \in \mathcal{W}$ has chosen this slot. These time slots are chosen by the tags in the set $\left\{\mathcal{T}_{r} \backslash\left\{\cup_{w \in \mathcal{W}} \mathcal{T}_{w}\right\}\right\}$. This suggests that the variable $Z$ can be used to estimate the number of tags in set $\left\{\mathcal{T}_{r} \backslash\left\{\cup_{w \in \mathcal{W}} \mathcal{T}_{w}\right\}\right\}$. Let $n_{\mathcal{W}}=\left|\left\{\cup_{w \in \mathcal{W}} \mathcal{T}_{w}\right\}\right|$ denote the number of tags that are in the zones of neighboring readers in $\mathcal{W}$. The following theorem characterizes the behavior of variable $Z$.

Theorem 1. The random variable $Z$ has a binomial distribution with mean $\mu_{z}$ for large values of $n_{r(\mathcal{W})}$ and $n_{\mathcal{W}}$ :

$$
\mu_{z}=f\left(1-\exp \left(-\frac{p n_{r(\mathcal{W})}}{f}\right)\right) \exp \left(-\frac{p n_{\mathcal{W}}}{f}\right)
$$

For large values of $f, Z$ can be approximated by a normal distribution with mean $\mu_{z}$ and variance $\sigma_{z}^{2}$ as

$$
\begin{aligned}
& \sigma_{z}^{2}=f \exp \left(-\frac{p n_{\mathcal{W}}}{f}\right)\left(1-\exp \left(-\frac{p n_{r(\mathcal{W})}}{f}\right)\right) \\
& \left(1-\exp \left(-\frac{p n_{\mathcal{W}}}{f}\right)\left(1-\exp \left(-\frac{p n_{r(\mathcal{W})}}{f}\right)\right)\right) .
\end{aligned}
$$

The proof of Theorem 1 can be found in Appendix A. Consider $z$ slots are empty in $\mathbf{v}_{w}, \forall w \in \mathcal{W}$, and nonempty in $\mathbf{v}_{r}$ after an interrogation process. $z$ is a sample of the random variable $Z$. By equating the expected value (i.e., $\mu_{z}$ in equation (6)) and the observed value $z$ of random variable $Z$, we can estimate the value of $n_{r(\mathcal{W})}$ which is denoted by $\tilde{n}_{r(\mathcal{W})}$ as

$$
\tilde{n}_{r(\mathcal{W})}=-\frac{f}{p} \ln \left(1-\frac{z}{f} \exp \left(\frac{p}{f} \tilde{n}_{\mathcal{W}}\right)\right) .
$$

Equation (8) is the exclusive estimator used to estimate $n_{r}(\mathcal{W})$ given the values of $z$ and $\tilde{n}_{\mathcal{W}}$. There are two approaches to estimate the value of $n_{\mathcal{W}}$. One can merge (via bit-wise OR) the interrogation vectors of readers in $\mathcal{W}$ and create one interrogation vector. Then, EZB algorithm can be used to estimate the value of $n_{\mathcal{W}}$ using this interrogation vector. Our MRTE algorithm which is presented in the next subsection can also be used to estimate value of $n_{\mathcal{W}}$. The following theorem characterizes the estimation error of the exclusive estimator.

Theorem 2. The probability density function (pdf) of $\tilde{n}_{r(\mathcal{W})}$ can be approximated by a normal distribution with mean $\mu_{\tilde{n}_{r(\mathcal{W})}}$ and variance $\sigma_{\tilde{n}_{r(\mathcal{W})}}^{2}$ as

$$
\begin{aligned}
\mu_{\tilde{n}_{r(\mathcal{W})}} & \approx n_{r(\mathcal{W})}, \\
\sigma_{\tilde{n}_{r(\mathcal{W})}}^{2} & \approx\left(\frac{1}{p} \exp \left(\frac{p n_{r(\mathcal{W})}}{f}\right) \exp \left(\frac{p n_{\mathcal{W}}}{f}\right)\right)^{2} \sigma_{z}^{2} .
\end{aligned}
$$

The proof of Theorem 2 can be found in Appendix B. In Theorem 2, we assume that there is no error in estimation of $n_{\mathcal{W}}$. We investigate the effect of this error on estimation of $n_{r(\mathcal{W})}$ by simulations in Section IV.

\section{B. Multiple-Reader Tag Estimation (MRTE) Algorithm}

In this section, we present the MRTE algorithm to estimate the total number of tags by using the exclusive estimator introduced in Section III-A. Algorithm 1 shows the MRTE

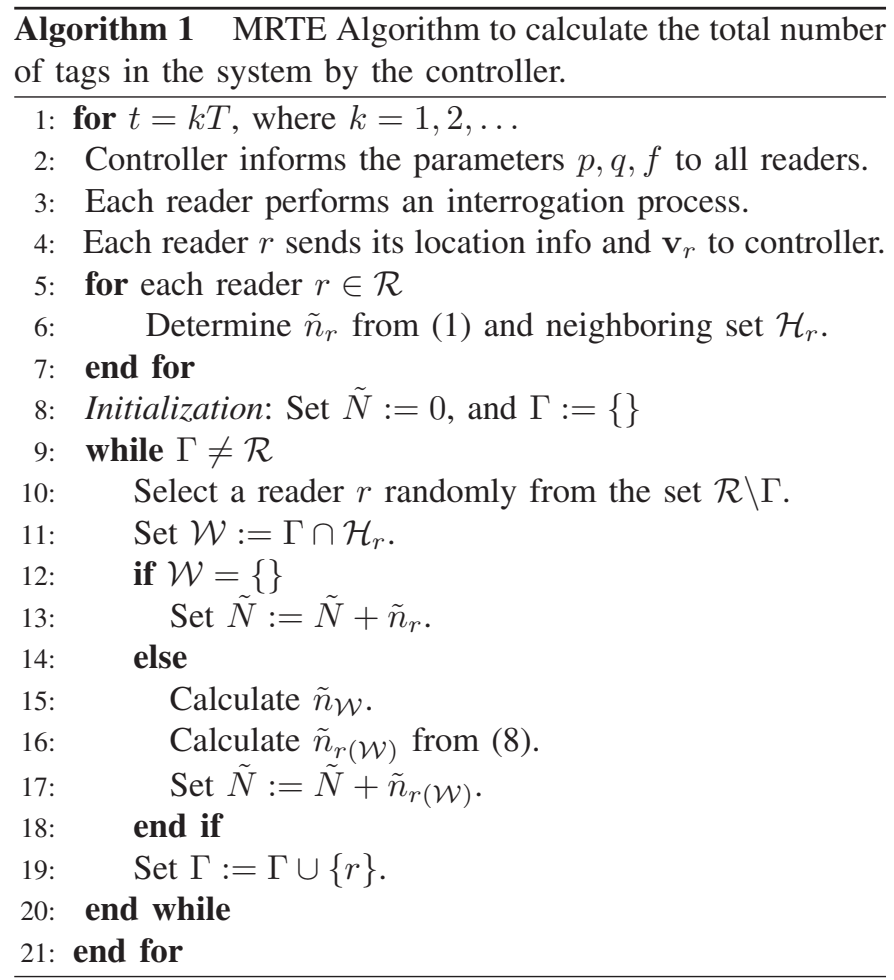

algorithm. The algorithm is invoked every $T$ time units (Step 1 ), where $T$ is a system parameter. When the algorithm is invoked, the controller informs the persistent probability $p$, frame size $f$, and random seed $q$ to all the readers (Step 2). Each reader $r \in \mathcal{R}$ then performs the interrogation process and sends the vector $\mathbf{v}_{r}$ (described in Section II.A) along with its location information to the controller (Steps 3 and 4). The controller can then estimate the number of tags within the zone of each reader $r$ by using (1). Based on the communication range of each reader and its location, $\mathcal{H}_{r}$, which is the set of neighboring readers of reader $r$, can also be determined (Step 6). The subsequent steps (Steps 9 - 19) are equivalent to applying equation (4) to estimate the total number of tags. In each step, a reader $r$ is selected randomly from the set $\mathcal{R} \backslash \Gamma$. The number of tags which is within the interrogation zone of $r$ but not in the zones of subset $\mathcal{W}$ is determined by using the exclusive estimator in (8). The estimated total number of tags $\tilde{N}$ is updated accordingly. The above procedure is repeated until the set $\Gamma$ is equal to the set of all readers $\mathcal{R}$.

Since the mean of error in estimation of variables $\tilde{n}_{r}$ and $\tilde{n}_{r(\mathcal{W})}$ is zero, the mean of error in estimation of the total number of tags is zero as well. The expected value of the variance of estimation error over different runs of algorithm is fixed. For pure random selection of readers, we calculate the expected value of the variance of estimation error over different runs of the algorithm. For reader $r \in \mathcal{R}$ with $\left|\mathcal{H}_{r}\right|$ neighbors, let $\mathcal{Q}_{r}$ denote the power set of $\mathcal{H}_{r}$. The power set of a set is the set of all subsets of that set. For exclusive estimator which is used to estimate $n_{r(\mathcal{W})}$, we have $\mathcal{W} \subset \mathcal{H}_{r}$. Therefore, $\mathcal{W}$ belongs to set $\mathcal{Q}_{r}$. During a run of the algorithm, when reader $r$ is selected, depending on the readers which have selected before, the number of tags within zone of reader $r$ 
may appear in various forms in $\tilde{N}$. For example it can be either $\tilde{N}:=\tilde{N}+\tilde{n}_{r}$ or $\tilde{N}:=\tilde{N}+\tilde{n}_{r(\mathcal{W})}$ for any $\mathcal{W} \in \mathcal{Q}$. For set $\mathcal{W} \in \mathcal{Q}$, the probability that $\tilde{N}$ contains $\tilde{n}_{r(\mathcal{W})}$ is equal to the probability that all readers in set $\mathcal{W}$ are selected before reader $r$ within an algorithm run. Assuming that the reader selection is purely random, this probability is equal to the probability that reader $r$ is selected at $k$ th step of the algorithm multiplied by the probability that all readers in set $\mathcal{W}$ are selected before that for $k=1, \ldots,|\mathcal{R}|$. Let $P_{n_{r(\mathcal{W})}}$ denote the probability that $n_{r(\mathcal{W})}$ is appeared in $\tilde{N}$. For reader $r$ and all $\mathcal{W} \in \mathcal{Q}_{r}$, this probability can be written as:

$$
\begin{aligned}
P_{n_{r(\mathcal{W})}} & =\sum_{k=1}^{|\mathcal{R}|}\left(P_{n_{r(\mathcal{W})}}^{k} P(r \text { selected as } k \text { th reader })\right) \\
& =\frac{1}{|\mathcal{R}|} \sum_{k=1}^{|\mathcal{R}|}\left(\frac{k-1}{|\mathcal{R}|}\right)^{|\mathcal{W}|}\left(1-\frac{k}{|\mathcal{R}|}\right)^{\left|\mathcal{H}_{r}\right|-|\mathcal{W}|}
\end{aligned}
$$

where $P_{n_{r(\mathcal{W})}}^{k}$ is the probability that from the neighbors of reader $r$, only readers in $\mathcal{W}$ are picked before $r$ and $r$ is chosen as $k$ th reader. When reader $r$ is selected within an algorithm run, with probability $P_{n_{r(\mathcal{W})}}$, we have $\mathcal{W}=\Gamma \bigcap \mathcal{H}_{r}, \forall \mathcal{W} \in$ $\mathcal{Q}_{r}$. The expected value for the variance of the error in estimating the total number of tags can be written as

$$
\tilde{\sigma}^{2}=\sum_{r \in \mathcal{R}} \sum_{\mathcal{W} \in \mathcal{Q}_{r}} P_{n_{r(\mathcal{W})}} \sigma_{\tilde{n}_{r(\mathcal{W})}}^{2}
$$

where $\sigma_{\tilde{n}_{r(\mathcal{W})}}^{2}$ is obtained from (10). If $\mathcal{W}=\emptyset$, then $\sigma_{\tilde{n}_{r(\mathcal{W})}}^{2}=$ $\sigma_{\tilde{n}_{r}}^{2}$ which is obtained from (3).

\section{Performance Evaluation}

We used MATLAB and developed a discrete-event RFID simulator. The analytical results are validated by comparing them with the results obtained from the simulator. Consider an RFID system with two readers with overlapping interrogation zones similar to one shown in Fig. 1. The interrogation zone of each reader has a range equal to $15 \mathrm{~m}$. The frame length $f$ is 400 time slots. The tags are randomly deployed in the interrogation zone of the readers. On average, the number of tags in the overlapped interrogation zone is $25 \%$ of the total number of tags in the interrogation zone of each reader.

We define the load factor as the number of tags in the interrogation zone of a reader divided by the frame length $f$. Fig. 2 shows the actual number of tags and the mean of estimated number of tags when the load factor is increased. The mean of estimated number of tags follows the actual number when the load factor is less than 3, 6, and 10 for persistent probability $p=1,0.5$, and 0.1 , respectively. These are the upper bounds for the operational range of the exclusive estimator and the approximations used in (9) and (10).

Fig. 3 shows the percentage of error in approximating the variance of the estimation error versus the load factor. The $\mathrm{y}$-axis is $\left|\left(\sigma^{2}-\tilde{\sigma}^{2}\right)\right| / \sigma^{2} \times 100 \%$, where $\sigma^{2}$ is the actual variance of the estimator error obtained via simulation and $\tilde{\sigma}^{2}$ is the approximated value from (12). Results show that the approximation error decreases when the persistent probability $p$ reduces. The approximation error is less than 35\%,10\%,

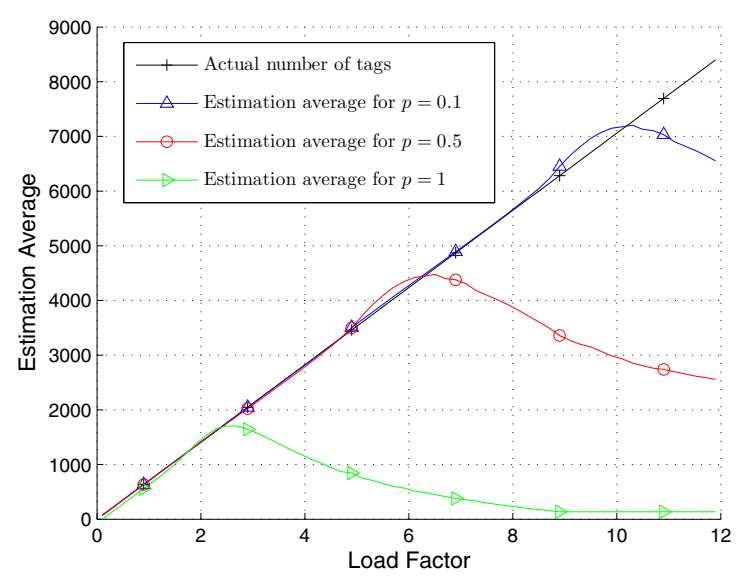

Fig. 2. Mean of estimated total number of tags in the system.

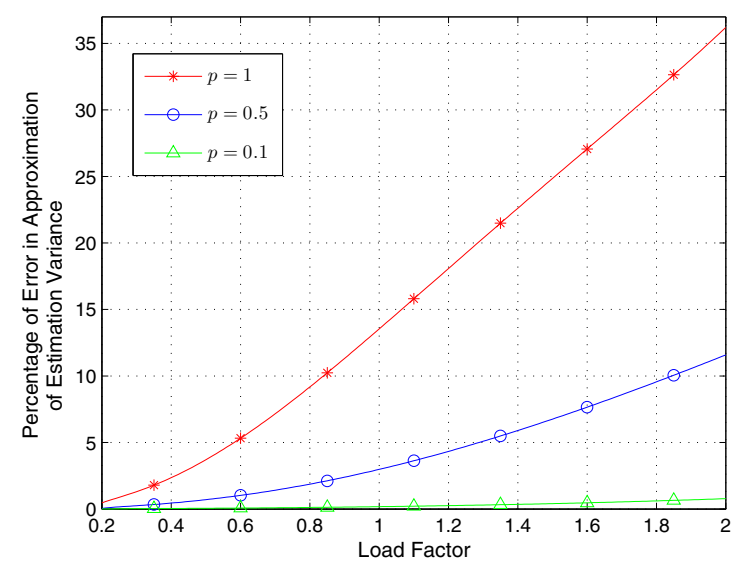

Fig. 3. Percentage of error in approximation of variance of estimation error.

and $2 \%$ when the load factor is less than two for $p=1,0.5$, and 0.1 , respectively.

We compare our proposed MRTE algorithm with two other estimation algorithms: maximum a posteriori tag estimation (MPTE) [11] and EZB [10] algorithms. The extension of the EZB algorithm for systems with multiple readers is introduced in [10]. We use the same approach and extend the MPTE algorithm for multi-reader RFID systems. We consider a twodimensional lattice of RFID readers where the vertical and horizontal distances of neighboring readers are $20 \mathrm{~m}$. The frame size $f$ is 400 time slots. The number of tags in the interrogation zone of each reader is 400 .

Fig. 4 shows the standard deviation of error in estimation of total number of tags for these three algorithms. It can be seen that the standard deviation of error in MRTE algorithm grows linearly with the number of readers while it increases exponentially in MPTE and EZB algorithms. For systems with small number of readers, MPTE and EZB perform slightly better than MRTE. However, MRTE outperforms these two algorithms when the number of readers is increased.

In the next experiment, the number of tags in the interrogation zone of a reader is varied while the frame size $f$ is fixed. Since all three algorithms give a zero mean of the estimation error, we determine the lower bound of the load factor such that the MTRE algorithm outperforms the MPTE and EZB algorithms in terms of a smaller estimation variance 


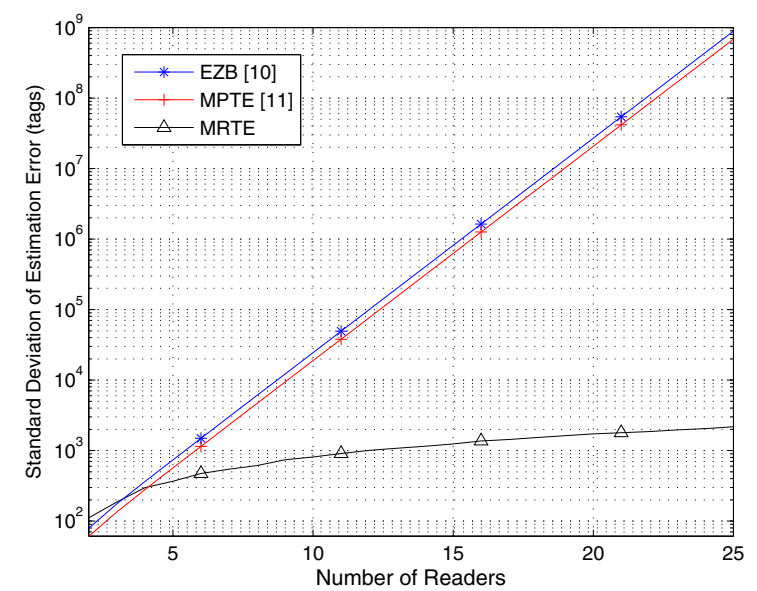

Fig. 4. Performance comparison of MRTE, EZB [10], and MPTE [11].

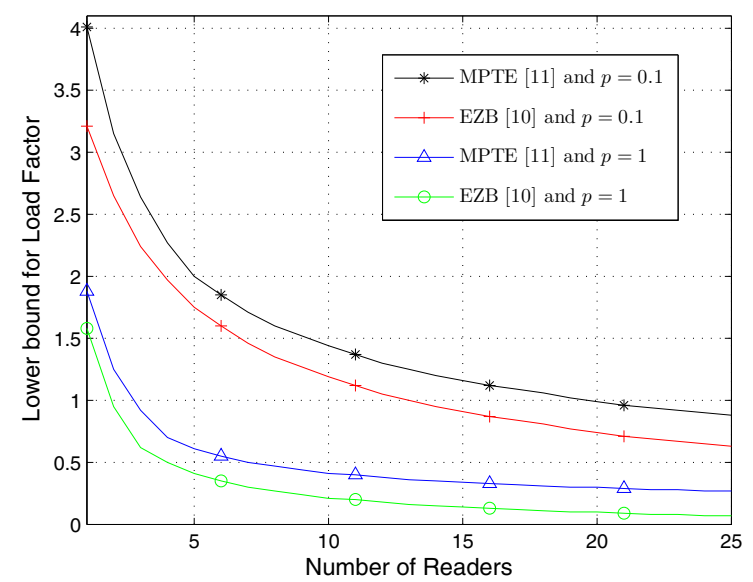

Fig. 5. Lower bound for the load factor for the better performance of MRTE compared to EZB [10] and MPTE [11].

$\tilde{\sigma}^{2}$. Fig. 5 shows this lower bound for different algorithms. As an example, results show that when $|\mathcal{R}|=5, p=1$, our proposed MRTE algorithm has a better performance than EZB and MPTE algorithms when the load factor is greater than 0.6. The lower bound decreases when the number of readers and number of tags increase in the system. Thus, our proposed MRTE algorithm is scalable.

In the last experiment, given the confidence interval $\beta$, we obtain the lower and upper bounds for the load factor such that for any load factor between these bounds, we have $\frac{\tilde{N}}{N} \times 100 \%$ $\in(100 \%-\beta, 100 \%+\beta)$ with probability greater than 0.95 . These bounds are shown in Fig. 6 where the confidence level $\beta$ is chosen as $80 \%, 85 \%$, and $90 \%$, respectively. As an example, for $\beta=90 \%$, results show that if the load factor is between two curves for the $90 \%$ confidence level, then the probability of having an estimated value $\tilde{N}$ within $\pm 10 \%$ range of the actual value $N$ is greater than or equal to 0.95 .

\section{CONCLUSIONS}

In this paper, we studied the problem of anonymous cardinality estimation in RFID systems with multiple readers. Each tag only transmits a portion of its ID when it is being queried by a reader. We proposed an exclusive estimator to estimate the number of tags which are exclusively located in the interrogation zone of a reader. We then proposed a

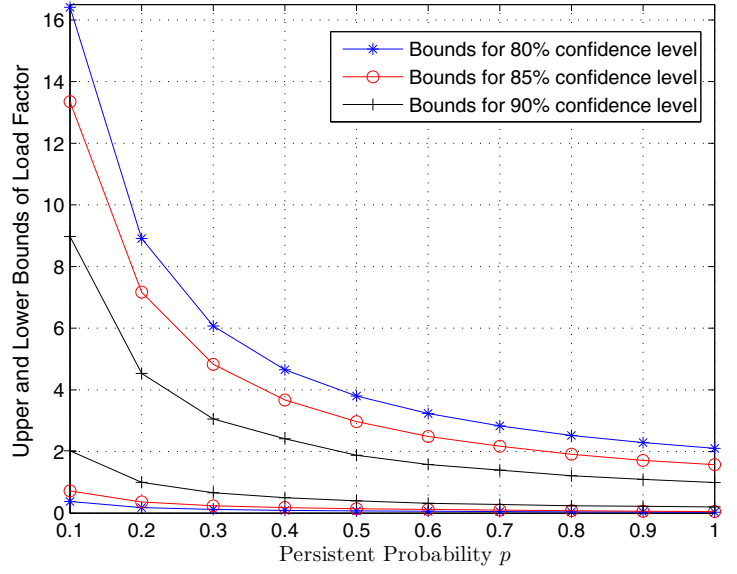

Fig. 6. Upper and lower bounds of the load factor for different confidence interval $\beta$ versus persistent probability $p$.

multiple-reader tag estimation (MRTE) algorithm which can accurately estimate the tag population anonymously using the query replies among different readers. We derived the probability density function of the estimation error and showed that it can be approximated as a normal distribution with zero mean. The accuracy of the model and the approximations are validated via simulations. For performance comparisons, results showed that the variance of estimation error for our proposed MRTE algorithm increases linearly with the number of readers while it increases exponentially for EZB [10] and MPTE [11] algorithms.

\section{A. Proof of Theorem 1}

\section{APPENDIX}

Let $\phi_{h}$ and $\psi_{h}$ denote the event that $h$ predetermined slots are nonempty in $\mathbf{v}_{r}$ and empty in $\mathbf{v}_{w}, \forall w \in \mathcal{W}$, respectively. Let $\theta_{h}$ denote the event that both events $\phi_{h}$ and $\psi_{h}$ occur. Since the events $\phi_{h}$ and $\psi_{h}$ are independent, we have

$$
\mathrm{P}\left(\theta_{h}\right)=\mathrm{P}\left(\phi_{h} \cap \psi_{h}\right)=\mathrm{P}\left(\phi_{h}\right) \mathrm{P}\left(\psi_{h}\right),
$$

where

$$
\begin{aligned}
\mathrm{P}\left(\phi_{h}\right)=\sum_{t=0}^{n_{r(\mathcal{W})}}\left(\mathrm{P}\left(\phi_{h} \mid t \text { tags pick these } h \text { slots }\right)\right. \\
\times \mathrm{P}(t \text { tags pick these } h \text { slots })) .
\end{aligned}
$$

The probability that $h$ time slots are non-empty if $t$ tags choose those slots is as follows [14, p. 92]:

$\mathrm{P}\left(\phi_{h} \mid t\right.$ tags pick these $h$ slots $)=\sum_{k=0}^{h}(-1)^{k}\left(\begin{array}{l}h \\ k\end{array}\right)\left(1-\frac{k}{f}\right)^{t}$.

Hence, we have

$$
\begin{aligned}
& \mathrm{P}\left(\phi_{h}\right)=\sum_{t=0}^{n_{r(\mathcal{W})}}\left(\sum_{k=0}^{h}(-1)^{k}\left(\begin{array}{l}
h \\
k
\end{array}\right)\left(1-\frac{k}{f}\right)^{t}\left(\begin{array}{c}
n_{r(\mathcal{W})} \\
t
\end{array}\right)\right. \\
& \left.\left(\frac{p h}{f}\right)^{t}\left(1-\frac{p h}{f}\right)^{\left(n_{r(\mathcal{W})}-t\right)}\right) \\
& =\sum_{k=0}^{h}(-1)^{k}\left(\begin{array}{l}
h \\
k
\end{array}\right)\left(1-\frac{p k}{f}\right)^{n_{r(\mathcal{W})}} \text {. }
\end{aligned}
$$


When $p k / f<1$ and $n_{r(\mathcal{W})}$ is large, $\left(1-\frac{p k}{f}\right)^{n_{r(\mathcal{W})}}$ can be approximated as $\exp \left(-\frac{p k n_{r(\mathcal{W})}}{f}\right)$. Thus, we have

$$
\begin{aligned}
\mathrm{P}\left(\phi_{h}\right) & \approx \sum_{k=0}^{h}\left(\begin{array}{l}
h \\
k
\end{array}\right)\left(-\exp \left(-\frac{p n_{r(\mathcal{W})}}{f}\right)\right)^{k} \\
& =\left(1-\exp \left(-\frac{p n_{r(\mathcal{W})}}{f}\right)\right)^{h} .
\end{aligned}
$$

We also have

$$
\mathrm{P}\left(\psi_{h}\right)=\prod_{w \in \mathcal{W}}\left(1-\frac{p h}{f}\right)^{n_{w}} \approx \exp \left(-\frac{p h n_{\mathcal{W}}}{f}\right) .
$$

Therefore, we can write $\mathrm{P}\left(\theta_{h}\right)$ as

$$
\mathrm{P}\left(\theta_{h}\right) \approx\left(1-\exp \left(-\frac{p n_{r(\mathcal{W})}}{f}\right)\right)^{h} \exp \left(-\frac{p n_{\mathcal{W}}}{f}\right)^{h} .
$$

For notation simplicity, we define

$$
\eta=\left(1-\exp \left(-\frac{p n_{r(\mathcal{W})}}{f}\right)\right) \exp \left(-\frac{p n_{\mathcal{W}}}{f}\right) .
$$

Let $S_{h}$ denote the summation of probability of all possible occurrences of $h$ events in a frame. This is equal to $S_{h}=$ $\left(\begin{array}{l}f \\ h\end{array}\right) P\left(\theta_{h}\right)$. Let $P_{h}$ denote the probability of having exactly $h$ slots nonempty in $\mathbf{v}_{r}$ and empty in $\mathbf{v}_{w}, \forall w \in \mathcal{W}$. This probability can be calculated using [14, p. 96] as follows:

$$
\begin{aligned}
P_{h} & =\sum_{m=h}^{f}(-1)^{m-h}\left(\begin{array}{c}
m \\
h
\end{array}\right) S_{m} \\
& =\sum_{m=h}^{f}(-1)^{m-h}\left(\begin{array}{c}
m \\
h
\end{array}\right)\left(\begin{array}{c}
f \\
m
\end{array}\right) \eta^{m} \\
& =\left(\begin{array}{l}
f \\
h
\end{array}\right) \sum_{m=h}^{f}(-1)^{m-h}\left(\begin{array}{c}
f-h \\
m-h
\end{array}\right) \eta^{m} \\
& =\left(\begin{array}{l}
f \\
h
\end{array}\right) \eta^{h} \sum_{m^{\prime}=0}^{f-h}(-\eta)^{m^{\prime}}\left(\begin{array}{c}
f-h \\
m^{\prime}
\end{array}\right) \\
& =\left(\begin{array}{c}
f \\
h
\end{array}\right) \eta^{h}(1-\eta)^{f-h}
\end{aligned}
$$

The probability in (16) is a binomial distribution with mean $\mu_{z}=f \eta$ and variance $\sigma_{z}^{2}=f \eta(1-\eta)$, where $\eta$ is defined in (15). When $f$ is large, the binomial distribution can be approximated by a normal distribution, with mean $\mu_{z}$ and variance $\sigma_{z}^{2}[15]$.

\section{B. Proof of Theorem 2}

We define a random variable $\gamma$ as $\gamma=z / f e^{p n_{\mathcal{W}} / f}$. Variable $\gamma$ is a normal random variable with mean and variance equal to $\mu_{\gamma}$ and $\sigma_{\gamma}^{2}$, respectively, as follows:

$$
\begin{gathered}
\mu_{\gamma}=\frac{1}{f} \exp \left(\frac{p n_{\mathcal{W}}}{f}\right) \mu_{z}, \\
\sigma_{\gamma}^{2}=\left(\frac{1}{f} \exp \left(\frac{p n_{\mathcal{W}}}{f}\right)\right)^{2} \sigma_{z}^{2} .
\end{gathered}
$$

For large values of $f$ and bounded $p n_{w} / f$, we have $\sigma_{\gamma}^{2}<<1$. Using Taylor series expansion, we can approximate equation (8) as

$$
\tilde{n}_{r(\mathcal{W})} \approx-\frac{f}{p}\left(\ln \left(1-\mu_{\gamma}\right)-\frac{\gamma-\mu_{\gamma}}{1-\mu_{\gamma}}\right) .
$$

Since variable $\gamma$ is a normal random variable, $\tilde{n}_{r(\mathcal{W})}$ can be approximated by a random variable with mean $\mu_{\tilde{n}_{r(\mathcal{W})}}$ and variance $\sigma_{\tilde{n}_{r(\mathcal{W})}}^{2}$ as

$$
\begin{aligned}
\mu_{\tilde{n}_{r(\mathcal{W})}} & \approx-\frac{f}{p} \ln \left(1-\mu_{\gamma}\right)=n_{r(\mathcal{W})} \\
\sigma_{\tilde{n}_{r(\mathcal{W})}}^{2} & \approx\left(\frac{f}{p\left(1-\mu_{\gamma}\right)}\right)^{2} \sigma_{\gamma}^{2}=\left(\frac{e^{\mu_{\kappa}}}{p\left(1-\mu_{\gamma}\right)}\right)^{2} \sigma_{z}^{2}
\end{aligned}
$$

where $\sigma_{z}^{2}$ is defined in equation (7). Consequently, the error in estimation of variable $\tilde{n}_{r(\mathcal{W})}$ has a normal distribution with zero mean and variance $\sigma_{\tilde{n}_{r(\mathcal{W})}}^{2}$.

\section{ACKNOWLEDGEMENT}

This research is supported by the Natural Sciences and Engineering Research Council (NSERC) of Canada under grant number STPGP 364962-08.

\section{REFERENCES}

[1] J. Myung and W. Lee, "Adaptive splitting protocols for RFID tag collision arbitration," in Proc. of ACM MobiHoc, Florence, Italy, May 2006.

[2] C. Floerkemeier, "Bayesian transmission strategy for framed Aloha based RFID protocols," in Proc. of IEEE Int'l Conf. on RFID, Grapevine, TX, Mar. 2007.

[3] Y.-C. Ko, S. Roy, J. R. Smith, H.-W. Lee, and C.-H. Cho, "An enhanced dynamic RFID multiple access protocol for fast inventory," in Proc. of IEEE Globecom, Washington, DC, Nov. 2007.

[4] J. S. Choi, H. Lee, D. W. Engels, and R. Elmasri, "Robust and dynamic bin slotted anti-collision algorithms in RFID systems," in Proc. of IEEE Int'l Conf. on RFID, Las Vegas, NV, Apr. 2008.

[5] C.-H. Quan, J.-C. Choi, G.-Y. Choi, and C.-W. Lee, "The Slotted-LBT: A RFID reader medium access scheme in dense reader environments," in Proc. of IEEE Int'l Conf. on RFID, Las Vegas, NV, Apr. 2008.

[6] J.-B. Eom, T.-J. Lee, R. Rietman, and A. Yener, "Efficient framed-slotted Aloha algorithm with pilot frame and binary selection for anti-collision of RFID tags," IEEE Commun. Letters, vol. 12, pp. 861-863, Nov. 2008.

[7] A. H. Mohsenian-Rad, V. Shah-Mansouri, V. Wong, and R. Schober, "Randomized multi-channel interrogation algorithm for large-scale RFID systems," in Proc. of IEEE Globecom, Honolulu, HI, Dec. 2009.

[8] EPCglobal, "Class 1 Generation 2 UHF air interface protocol standard, v. 1.1.0," Dec. 2005. [Online]. Available: http://www.epcglobalinc.org/standards/uhfc1g2

[9] M. Kodialam and T. Nandagopal, "Fast and reliable estimation schemes in RFID systems," in Proc. of ACM Mobicom, LA, CA, Sept. 2006.

[10] M. Kodialam, T. Nandagopal, and W. C. Lau, "Anonymous tracking using RFID tags," in Proc. of IEEE INFOCOM, Anchorage, Alaska, May 2007.

[11] W.-T. Chen, "An accurate tag estimate method for improving the performance of an RFID anticollision algorithm based on dynamic frame length ALOHA," IEICE Trans. on Automatic Science and Engineering, vol. 6, pp. 9-15, Jan. 2009.

[12] G. Maselli, C. Petrioli, and C. Vicari, "Dynamic tag estimation for optimizing tree slotted Aloha in RFID networks," in Proc. of ACM MSWiM, Vancouver, CA, Oct. 2008.

[13] C. Qian, H. Ngan, and Y. Liu, "Cardinality estimation for large-scale RFID systems," in Proc. of IEEE Percom, Galveston, Texas, Mar. 2008.

[14] W. Feller, An Introduction to Probability Theory and Its Application. John Wiley and Sons, 1968.

[15] I. Blake, An Introduction to Applied Probability. John Wiley and Sons, 1987. 